\title{
Study of effects of mutual inductance on power system simulation
}

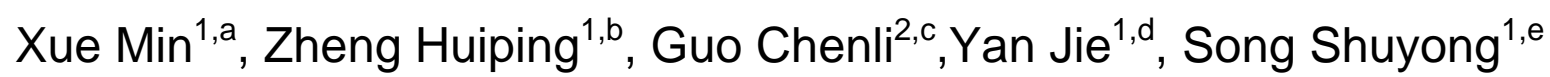

${ }^{1}$ State Grid Shanxi Electric Power Research Institute, Taiyuan 030001, China

\author{
${ }^{2}$ Shanxi Electric Power Exploration \& Design Institute of China Energy Engineering Group, Taiyuan \\ 030001, China \\ amig009@126.com, btyzhp01@163.com, c guochenli@126.com, dyanjie@sx.sgcc.com.cn, \\ esongshuyong@sx.sgcc.com.cn,
}

Keywords: mutual inductance; parameter; power system; operation

Abstract. The transmission lines of power system will produce mutual inductance in the actual operation. The mutual inductance makes parameter of transmission lines imbalance, which will lead to the operation of power system unbalanced. This paper makes a deep analysis in the reason of power system imbalance and illustrates the effects of mutual inductance on the power system simulation through the example.

\section{Introduction}

The three-phase voltage meeting at 120 degrees have the same amplitude in AC power system, which is called a three-phase balanced or symmetrical system. However, in actual operation, the power system is not completely balanced because of many unbalanced factors.To sum up, the factors causing phase unbalance in power system are three kinds: power system faults, parameter of elements in power system asymmetry and asymmetric load.This paper mainly conducts the research to the parameter of elements asymmetry, caused by mutual inductance,which will effect on power system simulation.

\section{The line parameters considering mutual inductance}

The instantaneous voltage value of three-phase system is:

$\left\{\begin{array}{l}u_{a}=U_{m} \sin (\omega t+\varphi) \\ u_{b}=U_{m} \sin \left(\omega t+\varphi-\frac{2 \pi}{3}\right) \\ u_{\mathrm{c}}=U_{m} \sin \left(\omega t+\varphi-\frac{4 \pi}{3}\right)\end{array}\right.$

The voltage Vector of three-phase system is:

$\left\{\begin{array}{l}\dot{U}_{A}=\dot{U} e^{j \varphi} \\ \dot{U}_{A}=\dot{U} e^{j\left(\varphi-\frac{2 \pi}{3}\right)} \\ \dot{U}_{A}=\dot{U} e^{j\left(\varphi-\frac{4 \pi}{3}\right)}\end{array}\right.$

The operator a is introduced to calculate easily:

$$
\begin{aligned}
& a=e^{j \frac{2 \pi}{3}}=-\frac{1}{2}+j \frac{\sqrt{3}}{2} \\
& a^{2}=e^{j \frac{4 \pi}{3}}=-\frac{1}{2}-j \frac{\sqrt{3}}{2} \\
& a^{3}=e^{j 2 \pi}=1
\end{aligned}
$$

The following formula can be drawn:

$1+a+a^{2}=0$

In order to study the effects of mutual inductance on unbalance of transmission line parameters,this paper adopts the method of symmetrical component,which will separate 
unsymmetric vector of three-phase system into three groups of symmetrical vector with different phase sequence.The zero sequence component is $A_{0}, B_{0}$ and $C_{0}$, the positive sequence component is $A_{1}, B_{1}$ and $C_{1}$, the negative sequence component is $A_{2}, B_{2}$ and $C_{2}$, the following formulas can be drawn:

$$
\begin{aligned}
& \left\{\begin{array}{l}
\dot{A}_{0} \\
\dot{B_{0}}=\dot{A}_{0} \\
\dot{C_{0}}=\dot{A_{0}}
\end{array}\right. \\
& \left\{\begin{array}{l}
\dot{A_{1}} \\
\dot{B_{1}}=a^{2} \dot{A}_{1} \\
\dot{C_{1}}=a \dot{A}_{1}
\end{array}\right. \\
& \left\{\begin{array}{l}
\dot{A_{2}} \\
\dot{B_{2}}=a \dot{A}_{2} \\
\dot{C}_{2}=a^{2} \dot{A}_{2}
\end{array}\right.
\end{aligned}
$$

The following equation are derived by above formulas:

$$
\begin{aligned}
& \left\{\begin{array}{l}
A=\dot{A}_{0}+\dot{A}_{1}+\dot{A}_{2} \\
B=A_{0}+a^{2} \dot{A}_{1}+a \dot{A}_{2} \\
\dot{C}=\dot{A}_{0}+a \dot{A}_{1}+a^{2} \dot{A}_{2}
\end{array}\right. \\
& \left\{\begin{array}{l}
\dot{A}_{0}=\frac{1}{3}(\dot{A}+\dot{B}+\dot{C}) \\
\dot{A}_{1}=\frac{1}{3}\left(\dot{A}+a \dot{B}+a^{2} \dot{C}\right) \\
\dot{A}_{2}=\frac{1}{3}\left(\dot{A}+a^{2} \dot{B}+a \dot{C}\right)
\end{array}\right.
\end{aligned}
$$

Three-phase transmission line with equilateral arrangement is shown in figure 1 , the current flowing in which depends on the values of voltage and reactance.

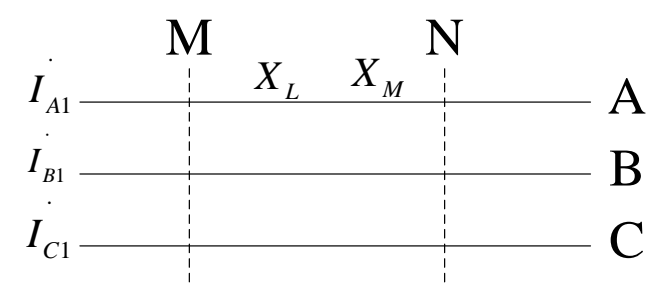

Fig.1 Schematic diagram of positive sequence reactance

The positive sequence component of current flowing in three-phase transmission line is:

$$
\left\{\begin{array}{l}
I_{A 1} \\
I_{B 1}=a^{2} I_{A 1} \\
I_{C 1}=a I_{A 1}
\end{array}\right.
$$

The positive sequence component of voltage between $\mathrm{M}$ and $\mathrm{N}$ in figure 1is: 


$$
\begin{aligned}
& U_{A 1}=j I_{A 1} X_{L}+j a^{2} I_{A 1} X_{M}+j a I_{A 1} X_{M} \\
& =j I_{A 1}\left[X_{L}+\left(a^{2}+a\right) X_{M}\right] \\
& =j I_{A 1}\left(X_{L}-X_{M}\right)
\end{aligned}
$$

The positive sequence component of impedance can be obtained as:

$$
X_{1}=X_{L}-X_{M}
$$

The $X_{L}$ is self-impedance of each phase, $X_{M}$ is mutual resistance which is produced by the other two phases. The negative sequence impedance is equal to the positive sequence impedance which is:

$X_{2}=X_{L}-X_{M}$

Similarly the zero sequence impedance can be deduce:

$$
X_{0}=X_{L}+2 X_{M}
$$

\section{The analysis of effects of mutual inductance on power system simulation}

The transmission lines adopts $\pi$ modle only considering the parameters of $\mathrm{R}, \mathrm{X}$ and $\mathrm{B} / 2$ in software BPA,which is used for simulation in power system. The effect of mutual inductance $\mathrm{M}$ on the power system simulation is not considered.This paper indicates the differences between considering mutual inductance or not considering mutual inductance in power system simulation through a typical small system. The simulation results in figure 2 and figure 3 show that the voltage of each substation increases slightly when mutual inductance of transmission line is under consideration.

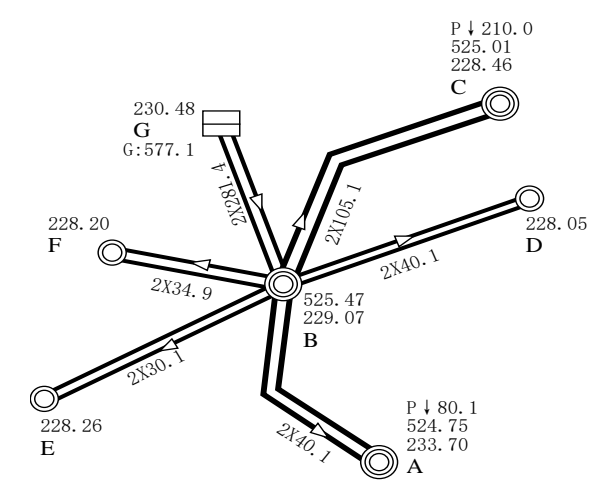

Fig.2 diagram of power flow distribution not considering mutual inductance

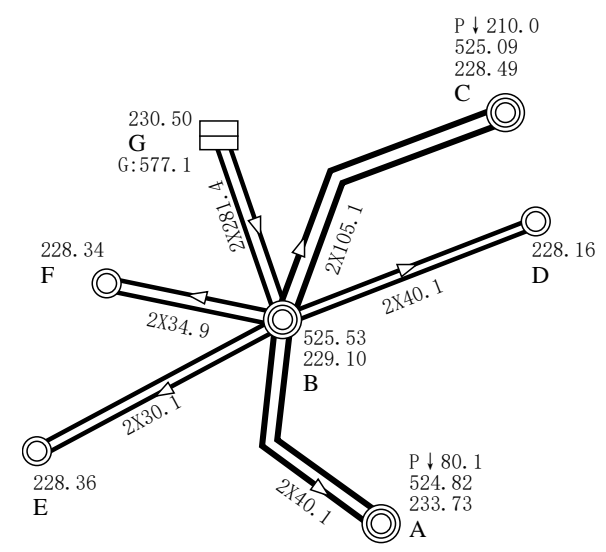

Fig.3 diagram of power flow distribution considering mutual inductance

\section{Conclusions}

Parameters of three-phase transmission line are unbalanced because of existence of mutual inductance, which will change the voltage of each substation and power flow distribution in power 
system. The electromagnetic interference is more complicated in the actual operation environment of transmission lines, this paper make a quantitative analysis through the simulation in typical small system, the conclusion of which has directive significance to actual operation of transmission lines.

\section{References}

[1]Li Gang,Feng Chenhu,Sun Jiwei,et al.Effects of mutual inductance of parallel transmission lines on longitudinal zero-sequence protection[J].North China Electric Power,2006,12:1-4.

[2]Hu Zhijian,Chen Yunping,Zhang Chengxue,et al.Measurement of mutual inductances parameters of long-Distance high-Voltage cable lines[J].High Voltage Engineering,2002,28(5):17-23.

[3]Wang Bo,XU Xidong,Fang Yudong.Fault location method for eliminating the influence of the mutual inductance of transmission lines in common corridor using one-terminal data[J].Power System Protection and Control,2011,39(14):1-6.

[4]Zhang Cong.Software Design for Calculation of Mutual Inductance Zero-sequence Parameters of EHV Transmission Lines[J].Guangdong Electric Power,2010,23(11):76-80.

[5]Liu Zunyi,Lu Ming,Lu Zhong-bin,et al.Power frequency measurement technology for UHV transmission lines and its application[J].Power System Technology,2009,33(10):59-62.

[6]Yuan Liangrong,Zhang Chi,Liu Wei.Measurement of mutual inductance between multiple-circuit lines of different voltage grades and analysis of its influence on relay protection setting [J]. Guangdong Electric Power,2007,20(11):22-25. 